ACS Chem Biol. 2016 July 15; 11(7): 1827-1833. doi:10.1021/acschembio.6b00324.

\title{
Hepatitis C Virus Selectively Alters the Intracellular Localization of Desmosterol
}

\author{
Valerie A. Villareal ${ }^{a}$, Dan Fu ${ }^{b, \dagger}$, Deirdre A. Costello ${ }^{a}$, Xiaoliang Sunney Xie $^{b}$, and Priscilla L. \\ Yang ${ }^{a,}$, \\ aDepartment of Microbiology and Immunobiology, Harvard Medical School, 77 Avenue Louis \\ Pasteur, Boston, MA 02115, U.S.A.. \\ ${ }^{b}$ Department of Chemistry and Chemical Biology, Harvard University, Cambridge, MA, U.S.A.
}

\section{Abstract}

\begin{abstract}
Hepatitis $\mathrm{C}$ virus (HCV) increases intracellular desmosterol without affecting the steady-state abundance of other sterols, and the antiviral activity of inhibitors of desmosterol synthesis is suppressed by the addition of exogenous desmosterol. These observations suggest a model in which desmosterol has a specific function, direct or indirect, in HCV replication and that HCV alters desmosterol homeostasis to promote viral replication. Here, we use stimulated Raman scattering (SRS) microscopy in combination with isotopically labeled sterols to show that HCV causes desmosterol to accumulate in lipid droplets that are closely associated with the viral NS5A protein and that are visually distinct from the broad distribution of desmosterol in mock-infected cells and the more heterogeneous and disperse lipid droplets to which cholesterol traffics. Localization of desmosterol in NS5A-associated lipid droplets suggests that desmosterol may affect $\mathrm{HCV}$ replication via a direct mechanism. We anticipate that SRS microscopy and similar approaches can provide much-needed tools to study the localization of specific lipid molecules in cellulo and in vivo.
\end{abstract}

\section{Abstract}

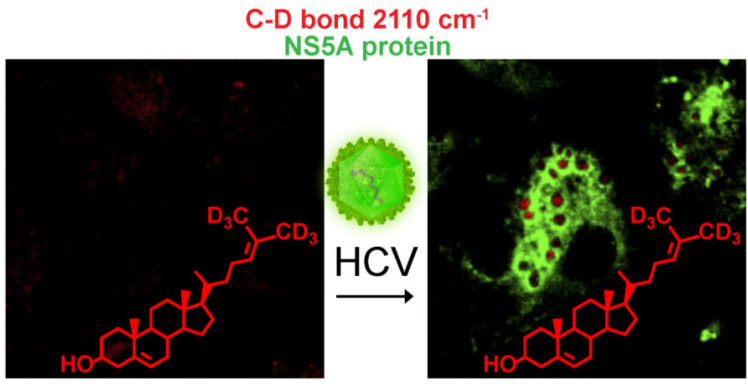

*Corresponding author. priscilla_yang@ @hms.harvard.edu.

†Present address: Department of Chemistry, University of Washington, Seattle, WA, U.S.A.

The authors declare no competing interests. 


\section{INTRODUCTION}

Many enveloped viruses require cellular lipids for every step in the viral replication cycle, including entry, expression and replication of the viral genome, and assembly and egress of progeny virions. Hepatitis $\mathrm{C}$ virus (HCV), which causes cirrhosis and liver cancer, chronically infects $130-150$ million people worldwide ${ }^{1}$ and has been shown to require lipids for each step in viral replication. Additionally, since HCV is a non-cytopathic virus, chronic infection leads to significant perturbations of cellular homeostasis, resulting in additional hallmark pathologies such as fatty liver disease (FLD) ${ }^{2}$. FLD, an accumulation of lipids in hepatocytes, demonstrates the profound ways in which viruses are able to alter not only metabolic flux but also to perturb steady-state cellular lipid metabolism. Although cellular lipids have been implicated in mediating viral entry, genome replication, assembly, and egress, we know relatively little of how lipid structure affects membrane function in these processes. A major limitation has been that we cannot easily study the structure, function, and localization of specific lipids involved in viral replication. Differences in mRNA or protein abundance for the relevant metabolic pathways can predict some changes in the steady-state abundance of classes of lipid molecules (e.g., fatty acids, triglycerides), but cannot provide the molecular structure of the specific lipid molecules present. Advances in mass spectrometry have made high-resolution lipidomic analyses to detect steady-state changes in lipid abundance increasingly routine. While powerful, extraction of the lipidome requires lysis of cells or membranes, a process that forfeits any information regarding spatial distribution of the lipid being monitored. One solution to this problem has been the conjugation of specific lipids to cell-permeable fluorophores to allow imaging of lipid localization in cellulo. This approach is limited by the potential for fluorophores, which are comparable in size to most lipid molecules, to alter the functional properties of the lipid to which they are conjugated. Likewise, although sterol-binding proteins fused to fluorescent proteins and natural products (e.g., perfringolysin $\mathrm{O}$ and filipins, respectively) can be used to visualize sterols, they generally cannot be used to distinguish one sterol from another ${ }^{3,4}$. Thus, although it is widely accepted that lipid bilayers are essential platforms for the chemical reactions that comprise viral replication, direct visualization of a specific lipid molecule in a viral process has remained challenging.

Lipid droplets (LDs) are cellular organelles that store neutral lipids (e.g., triacylglycerols and sterol esters) surrounded by a phospholipid monolayer. Recently, it was discovered that $\mathrm{HCV}$ increases LD formation, and LD were shown to be important platforms for the assembly of nascent $\mathrm{HCV}$ viral particles ${ }^{5-8}$. The $\mathrm{HCV}$ core protein, a structural component of virions, and the nonstructural protein 5A (NS5A) have been shown to interact with LDs, thereby forming a ring around the phospholipid layer of LD in HCV-infected cells ${ }^{5}$, 9-12. This is believed to provide temporal control over viral assembly by sequestering the core protein until sufficient copies of the viral RNA genome have been synthesized to allow productive assembly. The molecular mechanisms underlying these phenomena remain the subject of much investigation and whether specific lipids are mechanistically important remains largely unknown.

We recently discovered that replication of HCV clone JFH1 (HCV JFH1) in cell culture causes a 10-fold or greater increase in the steady-state abundance of desmosterol, the 
penultimate intermediate in the Bloch branch of cholesterol biosynthesis (Fig. 1) without affecting the steady-state abundance of cholesterol or other late stage sterols ${ }^{13}$. This increase in steady-state desmosterol has functional significance for $\mathrm{HCV}$ replication: $\mathrm{HCV}$ is inhibited by pharmacological inhibition or RNAi-mediated depletion of 7dehydrocholesterol reductase (DHCR7), the enzyme that directly catalyzes synthesis of desmosterol; moreover, this antiviral effect is reversed upon the addition of exogenous desmosterol ${ }^{13}$. While the effects of HCV JFH1 on desmosterol homeostasis and of desmosterol on HCV JFH1 replication were evident in these experiments, they provided no information regarding the intracellular distribution of desmosterol and whether it affects $\mathrm{HCV}$ via direct or indirect mechanisms. To investigate these possibilities, we sought methods to determine whether desmosterol accumulates at specific sites within the HCVinfected cell and, if so, if these sites are associated with other components of the viral replication machinery.

\section{RESULTS AND DISCUSSION}

Conventional imaging of sterols by fluorescence microscopy has been achieved through conjugation of the sterol to a fluorophore, such as BODIPY ${ }^{14-18}$. The aforementioned caveat to this approach is that this modification can affect the physicochemical properties of the sterol and may complicate its use as a tool to assess lipid localization and function in cell biological experiments. Recent advances in fluorophore-free optical microscopy methods, such as Raman microscopy ${ }^{19}$, provide a powerful alternative. In Raman microscopy, the intrinsic vibration of chemical bonds is detected, thereby simplifying the analysis of macromolecules since nucleic acids, lipids, and proteins exhibit different vibrational frequencies. Elegant examples of this include imaging of the $\mathrm{C}-\mathrm{H}$ stretch prevalent in lipids to monitor lipid droplet dynamics and their association with HCV core protein and RNA by coherent anti-Stokes Raman scattering (CARS) microscopy ${ }^{7,20}$.

To examine the specific effect of HCV on desmosterol (versus other lipids) in cellulo, we used stimulated Raman scattering (SRS) microscopy, a sensitive approach in which the frequencies of two lasers (Pump beam and Stokes beam) are tuned to match the molecular vibrational frequency of the chemical bond of interest. The signal intensity is proportional to the concentration of the chemical bond being detected ${ }^{21,22}$. SRS microscopy can detect and delineate lipids (tuned to $\mathrm{CH}_{2}$ stretches), unique bond arrangements, and proteins (tuned to $\mathrm{NH}_{2}$ stretches). By tuning the dual lasers to the vibrational frequencies of bonds that include lowly populated isotopes, signals from molecules enriched with these isotopes can be acquired separately from molecules that contain conventional bonds. For example, although deuterium $\left({ }^{2} \mathrm{H}\right.$ or $\left.\mathrm{D}\right)$, a stable isotope of hydrogen, behaves virtually identically to hydrogen $\left({ }^{1} \mathrm{H}\right)$ in biological systems, the $\mathrm{C}_{-}^{2} \mathrm{H}$ (carbon-deuterium or C-D) bond stretch at $2110 \mathrm{~cm}^{-1}$ is distinct from that of $\mathrm{C}^{-1} \mathrm{H}$ at $2854 \mathrm{~cm}^{-1}$. For clarity, C-D will refer to $\mathrm{C}-{ }^{2} \mathrm{H}$ and $\mathrm{C}-\mathrm{H}$ will refer to $\mathrm{C}^{1}{ }^{1} \mathrm{H}$. Tuning of the SRS microscope to the $\mathrm{C}-\mathrm{D}$ bond wave number thus allows selective visualization of a ${ }^{2} \mathrm{H}$-labelled metabolite or drug while avoiding the potential for functional perturbations that arise with the use of fluorophore-conjugated metabolites ${ }^{23}$.

To utilize this approach to study the effects of HCV on intracellular desmosterol, we first confirmed that deuterated desmosterol (Des-D6, Fig. 1a) functionally substitutes for 
desmosterol in our previously reported virological assays ${ }^{13}$ by showing that Des-D6 rescues replication of HCV JFH1 when desmosterol synthesis is blocked by AY9944, a pharmacological inhibitor of DHCR7 (Fig. S1). Next, we examined the extent to which exogenously added Des-D6 is converted to cholesterol in cellulo since this reaction would affect interpretation of the SRS microscopy experiments. By quantitative liquid chromatography - mass spectrometry (LC-MS) analysis of whole-cell lipidomes, we found that Des-D6 added to Huh7.5 cells is indeed converted to cholesterol (Fig. 1b). The presence of triparanol, a small molecule inhibitor of 24-dehydrocholesterol reductase (DHCR24) 24,25 , in the culture medium effectively blocks this and prevents metabolism of Des-D6 to cholesterol (Fig. 1b). To corroborate this in intact cells, we performed hyperspectral SRS microscopy on individual puncta in the HCV JFH1-infected Huh7.5 cell monolayers to which we had added Des-D6 in the presence and absence of triparanol. Comparison of these spectra with those taken for deuterated sterol standards (Fig. 1c) confirmed that puncta in the HCV JFH1-infected cell monolayer without triparanol (Fig. 1d) most closely resemble the spectrum for the Chol-D7 standard while puncta formed in the presence of triparanol more closely resemble the spectrum of the Des-D6 standard (Fig. 1e). Together, these observations demonstrated our ability to distinguish Des-D6 from Chol-D7 and established conditions under which we can monitor the localization of Des-D6 specifically by preventing its conversion to Chol-D6.

To examine the subcellular localization of Des-D6 in cells, we infected Huh7.5 cells with HCV JFH1 and added exogenous Des-D6 and triparanol with the cell culture medium followed by analysis of the localization of Des-D6 at 48 hours post-infection. We initially isolated lipid droplets by ultracentrifugation in order to examine their sterol content; however, lipid mixing of fractions compromised this analysis (data not shown). Likewise, we found that fillipin III was unsuitable for monitoring localization of the exogenously added desmosterol or Des-D6 because it stains both cholesterol and desmosterol (Fig. S2). As an alternative, we fixed the cell monolayers and performed immunofluorescence staining for the HCV NS5A protein, then imaged the samples via fluorescence and SRS microscopy to visualize NS5A and Des-D6, respectively. To visualize the C-D stretch, we tuned the lasers such that their difference was $2110 \mathrm{~cm}^{-1}$. This allowed detection of free and esterified forms of Des-D6 due to the location of the deuterium atoms on C24 and C25. Mock-infected cells exhibit a diffuse distribution of Des-D6 (Figs. 2a and S3a). In contrast, in cells infected with HCV JFH1, Des-D6 localizes in distinct puncta that are closely associated with NS5A (Figs. $2 \mathrm{~b}$ and $\mathrm{S} 3 \mathrm{~b}$ ) and that are reminiscent of the LDs observed in hepatoma cells replicating $\mathrm{HCV}^{6}$.

Since HCV has previously been shown to induce and increase LD formation, we questioned whether localization of desmosterol in these puncta could be due to increased lipid droplet biogenesis. To examine this possibility, we induced LD formation in uninfected Huh7.5 cells via an independent mechanism. Oleic acid activates a signalling cascade involving phosphoinositide 3-kinase, AKT, and phospholipase D. This leads to incorporation of endogenous lipids into LDs, induction of triglyceride synthesis, and incorporation of oleic acid itself into LDs ${ }^{26}$. Nile red staining of uninfected Huh7.5 cells treated with exogenous desmosterol revealed disperse staining (Fig. S4) that resembled the diffuse signal for Des-D6 observed in uninfected Huh7.5 cells by SRS microscopy (Fig. 2a). As expected, the addition 
of oleic acid increased intracellular lipid content due to induction of LD biogenesis even in the absence of HCV or exogenous sterols (Fig. S4). The addition of exogenous cholesterol or desmosterol increased Nile red staining in uninfected cells treated with oleic acid (Fig. S4), indicating that these sterols are trafficked to LDs despite the absence of NS5A or other viral proteins and RNA. To support the interpretation that exogenously added Des-D6 colocalizes specifically with LDs in $\mathrm{HCV}$-infected and oleic acid-treated cells, we additionally imaged the localization of $\mathrm{C}-\mathrm{H}$ bonds present in endogenous lipids and in exogenously added unlabelled desmosterol as well as the localization of C-D bonds present in exogenously added Des-D6. As was previously reported ${ }^{7,20}$, imaging of the C-H stretch $\left(2854 \mathrm{~cm}^{-1}\right)$ allowed detection of LDs, which we observed in the presence of oleic acid as well as HCV JFH1 (Fig. 3). Tuning of the SRS microscope to the C-D stretch $\left(2110 \mathrm{~cm}^{-1}\right)$ revealed little to no background signal in samples lacking Des-D6 (Fig. 3a), demonstrating that $\mathrm{C}-\mathrm{H}$ bonds contribute little to no background signal when the SRS microscope is tuned to detect C-D bonds. In contrast, there was abundant signal for Des-D6 that co-localized substantially with the C-H stretch $\left(2854 \mathrm{~cm}^{-1}\right)$ in oleic acid-treated and HCV JFH1-infected cells (Figs. $3 \mathrm{~b}$ and c). These observations confirm the trafficking of Des-D6 to lipid droplets in both HCV JFH1-infected and oleic acid-treated cells. This may suggest that the increased presence of LDs is sufficient to account for the difference in localization of Des-D6 in HCVinfected versus mock-infected cells. We note, however, that the appearance of Des-D6 LDs appears more heterogeneous in cells treated with oleic acid compared to those infected with HCV JFH1 (compare Figs. 3b versus 3c). It is presently unclear whether this difference is due to the presence of more LDs in the oleic acid-treated sample or whether there is a qualitative difference in the LDs induced by these two mechanisms.

We next considered another aspect of lipid specificity by asking whether HCV has a similar effect on the localization of other sterols, in particular, 7-dehydrodesmosterol and cholesterol, the immediate upstream and downstream sterols in the pathway (Fig. 1a). Huh7.5 cells were infected with HCV JFH1 and pretreated with triparanol prior to the addition of deuterated 7-dehydrodesmosterol (7DHD-D6) or deuterated cholesterol (CholD7). At 48 hours post-infection, NS5A and the C-D bond were visualized by two-photon fluorescence and SRS microscopy, respectively. Signal for the C-D stretch present in 7DHDD6 localized in lipid droplets with punctate morphology similar to those observed for DesD6 (Fig. S5). This indicates that HCV causes re-localization of 7DHD-D6 to these sites and/or that 7DHD-D6 is converted to Des-D6 and then trafficked to lipid droplets. In contrast, Chol-D7 localized in puncta that appeared smaller and more heterogeneous albeit more numerous than those observed for Des-D6 and 7DHD-D6 (Figs. 4 and S5). This qualitative impression was reinforced by analysis of the C-D bond signal intensities plotted versus distance across the image, which revealed more consistent diameter and signal intensity of Des-D6 puncta versus Chol-D7 puncta (Fig. 4). While the molecular basis for HCV's differential effects on the localization of desmosterol and cholesterol are still unknown, the differences observed suggest that the subtle structural difference between these two sterols - an alkene between carbons 24 and 25 -- is functionally significant. Likewise, although work to elucidate the mechanism(s) whereby HCV causes a selective increase in steady-state intracellular desmosterol is on-going, these results suggest that sequestration of desmosterol in lipid droplets may be a significant contributing factor. 
Although there is growing appreciation for how cell-derived membranes support viral replication, experiments that examine how lipid structure affects membrane properties and function in these processes have been lacking. A major limitation has been the absence of tools analogous to those used to study protein and nucleic acid structure, function, and localization. Here, we show that SRS microscopy can be used in combination with isotopically labelled sterols and inhibitors of sterol biosynthesis to monitor the subcellular localization of a specific sterol. We previously showed that HCV JFH1 induces a ten-fold or greater increase in intracellular desmosterol without affecting intracellular levels of cholesterol or other late-stage sterols ${ }^{13}$. This perturbation of steady-state desmosterol appears to be important for viral replication since pharmacological inhibition or RNAimediated depletion of DHCR7 causes a severe inhibition of HCV that is rescued upon the addition of exogenous desmosterol ${ }^{13}$. These observations have suggested a model in which HCV alters desmosterol homeostasis to promote viral replication. The results we report here strengthen this model in the following ways. First, Des-D6 localizes in HCV-induced lipid droplets that are visually distinct from both the broad distribution of Des-D6 in mockinfected cells and the more heterogeneous lipid droplets in which Chol-D7 accumulates. This provides independent evidence that HCV affects intracellular desmosterol specifically. Second, localization of Des-D6 in lipid droplets induced by oleic acid in the absence of $\mathrm{HCV}$ suggests that lipid droplet formation may be sufficient to change desmosterol localization. While it is tempting to conclude that endogenously synthesized desmosterol accumulates in HCV-infected cells because it is sequestered in HCV-induced lipid droplets, additional experiments are needed to determine whether there is a causal relationship between these events. Third, Des-D6's localization in NS5A-associated lipid droplets is consistent with the idea that desmosterol may directly affect $\mathrm{HCV}$ replication. A critical next step is establishing experimental approaches that allow interrogation of how desmosterol's subtle structural difference from cholesterol may result in functional differences on the surface of or within the lipid droplet as well as functional differences in other viral membranes and their associated viral processes. We anticipate that SRS microscopy and similar approaches can provide much-needed tools to study the localization of specific lipid molecules in cellulo and in vivo. When used in combination with pharmacological tools, this platform provides a non-invasive approach for interrogating how lipid structure affects subcellular localization relative to viral processes as well as other functional properties relevant to viral replication.

\section{METHODS}

Detailed descriptions of experimental procedures and reagents are provided in Supporting Information.

\section{Supplementary Material}

Refer to Web version on PubMed Central for supplementary material.

\section{ACKNOWLEDGEMENTS}

The authors are thankful for support from NIH R01AI076442 and Diversity Supplement R01AI076442-S1 (PLY, VAV), NIH F32AI106178 (VAV), John and Virginia Kaneb Fellowship (PLY), and Department of Energy grant DE- 
SC0012411 (XSX). We thank Dr. Charles Rice (Rockefeller University via Apath LLC) for Huh7.5, JFH1, and monoclonal antibody 9E10; Dr. Takaji Wakita (National Institute of Infectious Diseases, Japan) for JFH1; and Dr. Jon Clardy and the Harvard Medical School Analytical Chemistry Core for use of mass spectrometers.

\section{REFERENCES}

[1]. World Health Organization, M. C.. Hepatitis C. Jul. 2015

[2]. Lonardo A, Adinolfi LE, Restivo L, Ballestri S, Romagnoli D, Baldelli E, Nascimbeni F, Loria P. Pathogenesis and significance of hepatitis $\mathrm{C}$ virus steatosis: an update on survival strategy of a successful pathogen. World J Gastroenterol. 2014; 20:7089-7103. [PubMed: 24966582]

[3]. Clejan S, Bittman R. Distribution and movement of sterols with different side chain structures between the two leaflets of the membrane bilayer of mycoplasma cells. J Biol Chem. 1984; 259:449-455. [PubMed: 6706946]

[4]. Nelson LD, Johnson AE, London E. How interaction of perfringolysin O with membranes is controlled by sterol structure, lipid structure, and physiological low $\mathrm{pH}$ : insights into the origin of perfringolysin O-lipid raft interaction. J Biol Chem. 2008; 283:4632-4642. [PubMed: 18089559]

[5]. Barba G, Harper F, Harada T, Kohara M, Goulinet S, Matsuura Y, Eder G, Schaff Z, Chapman MJ, Miyamura T, Brechot C. Hepatitis $\mathrm{C}$ virus core protein shows a cytoplasmic localization and associates to cellular lipid storage droplets. Proc Natl Acad Sci U S A. 1997; 94:1200-1205. [PubMed: 9037030]

[6]. Miyanari Y, Atsuzawa K, Usuda N, Watashi K, Hishiki T, Zayas M, Bartenschlager R, Wakita T, Hijikata M, Shimotohno K. The lipid droplet is an important organelle for hepatitis $\mathrm{C}$ virus production. Nat Cell Biol. 2007; 9:1089-1097. [PubMed: 17721513]

[7]. Lyn RK, Kennedy DC, Stolow A, Ridsdale A, Pezacki JP. Dynamics of lipid droplets induced by the hepatitis C virus core protein. Biochem Biophys Res Commun. 2010; 399:518-524. [PubMed: 20678475]

[8]. Li Q, Pene V, Krishnamurthy S, Cha H, Liang TJ. Hepatitis C virus infection activates an innate pathway involving IKK-alpha in lipogenesis and viral assembly. Nat Med. 2013; 19:722-729. [PubMed: 23708292]

[9]. Hope RG, Murphy DJ, McLauchlan J. The domains required to direct core proteins of hepatitis C virus and GB virus-B to lipid droplets share common features with plant oleosin proteins. J Biol Chem. 2002; 277:4261-4270. [PubMed: 11706032]

[10]. McLauchlan J, Lemberg MK, Hope G, Martoglio B. Intramembrane proteolysis promotes trafficking of hepatitis C virus core protein to lipid droplets. EMBO J. 2002; 21:3980-3988. [PubMed: 12145199]

[11]. Boulant S, Montserret R, Hope RG, Ratinier M, Targett-Adams P, Lavergne JP, Penin F, McLauchlan J. Structural determinants that target the hepatitis $C$ virus core protein to lipid droplets. J Biol Chem. 2006; 281:22236-22247. [PubMed: 16704979]

[12]. Boulant S, Targett-Adams P, McLauchlan J. Disrupting the association of hepatitis C virus core protein with lipid droplets correlates with a loss in production of infectious virus. The Journal of general virology. 2007; 88:2204-2213. [PubMed: 17622624]

[13]. Rodgers MA, Villareal VA, Schaefer EA, Peng LF, Corey KE, Chung RT, Yang PL. Lipid metabolite profiling identifies desmosterol metabolism as a new antiviral target for hepatitis $\mathrm{C}$ virus. J Am Chem Soc. 2012; 134:6896-6899. [PubMed: 22480142]

[14]. Craig IF, Via DP, Mantulin WW, Pownall HJ, Gotto AM Jr. Smith LC. Low density lipoproteins reconstituted with steroids containing the nitrobenzoxadiazole fluorophore. J Lipid Res. 1981; 22:687-696. [PubMed: 7276743]

[15]. Puri V, Watanabe R, Dominguez M, Sun X, Wheatley CL, Marks DL, Pagano RE. Cholesterol modulates membrane traffic along the endocytic pathway in sphingolipid-storage diseases. Nat Cell Biol. 1999; 1:386-388. [PubMed: 10559968]

[16]. Wiegand V, Chang TY, Strauss JF 3rd, Fahrenholz F, Gimpl G. Transport of plasma membranederived cholesterol and the function of Niemann-Pick C1 Protein. FASEB J. 2003; 17:782-784. [PubMed: 12594172]

[17]. Li Z, Mintzer E, Bittman R. First synthesis of free cholesterol-BODIPY conjugates. J Org Chem. 2006; 71:1718-1721. [PubMed: 16468832] 
[18]. Huang H, McIntosh AL, Atshaves BP, Ohno-Iwashita Y, Kier AB, Schroeder F. Use of dansylcholestanol as a probe of cholesterol behavior in membranes of living cells. J Lipid Res. 2010; 51:1157-1172. [PubMed: 20008119]

[19]. Cheng JX, Xie XS. Vibrational spectroscopic imaging of living systems: An emerging platform for biology and medicine. Science. 2015; 350:aaa8870. [PubMed: 26612955]

[20]. Nan X, Tonary AM, Stolow A, Xie XS, Pezacki JP. Intracellular imaging of HCV RNA and cellular lipids by using simultaneous two-photon fluorescence and coherent anti-Stokes Raman scattering microscopies. Chembiochem. 2006; 7:1895-1897. [PubMed: 17068839]

[21]. Freudiger CW, Min W, Saar BG, Lu S, Holtom GR, He C, Tsai JC, Kang JX, Xie XS. Label-free biomedical imaging with high sensitivity by stimulated Raman scattering microscopy. Science. 2008; 322:1857-1861. [PubMed: 19095943]

[22]. Ozeki Y, Dake F, Kajiyama S, Fukui K, Itoh K. Analysis and experimental assessment of the sensitivity of stimulated Raman scattering microscopy. Opt Express. 2009; 17:3651-3658. [PubMed: 19259205]

[23]. Fu D, Yu Y, Folick A, Currie E, Farese RV Jr. Tsai TH, Xie XS, Wang MC. In vivo metabolic fingerprinting of neutral lipids with hyperspectral stimulated Raman scattering microscopy. J Am Chem Soc. 2014; 136:8820-8828. [PubMed: 24869754]

[24]. Carver MJ, Freeman JG, Smith JA. Evaluation of a cholesterol-synthesis inhibitor (triparanol). Ann Intern Med. 1960; 53:462-467. [PubMed: 13691207]

[25]. Popjak G, Meenan A, Parish EJ, Nes WD. Inhibition of cholesterol synthesis and cell growth by 24(R,S),25-iminolanosterol and triparanol in cultured rat hepatoma cells. J Biol Chem. 1989; 264:6230-6238. [PubMed: 2703486]

[26]. Rohwedder A, Zhang Q, Rudge SA, Wakelam MJ. Lipid droplet formation in response to oleic acid in Huh-7 cells is mediated by the fatty acid receptor FFAR4. J Cell Sci. 2014; 127:31043115. [PubMed: 24876224] 

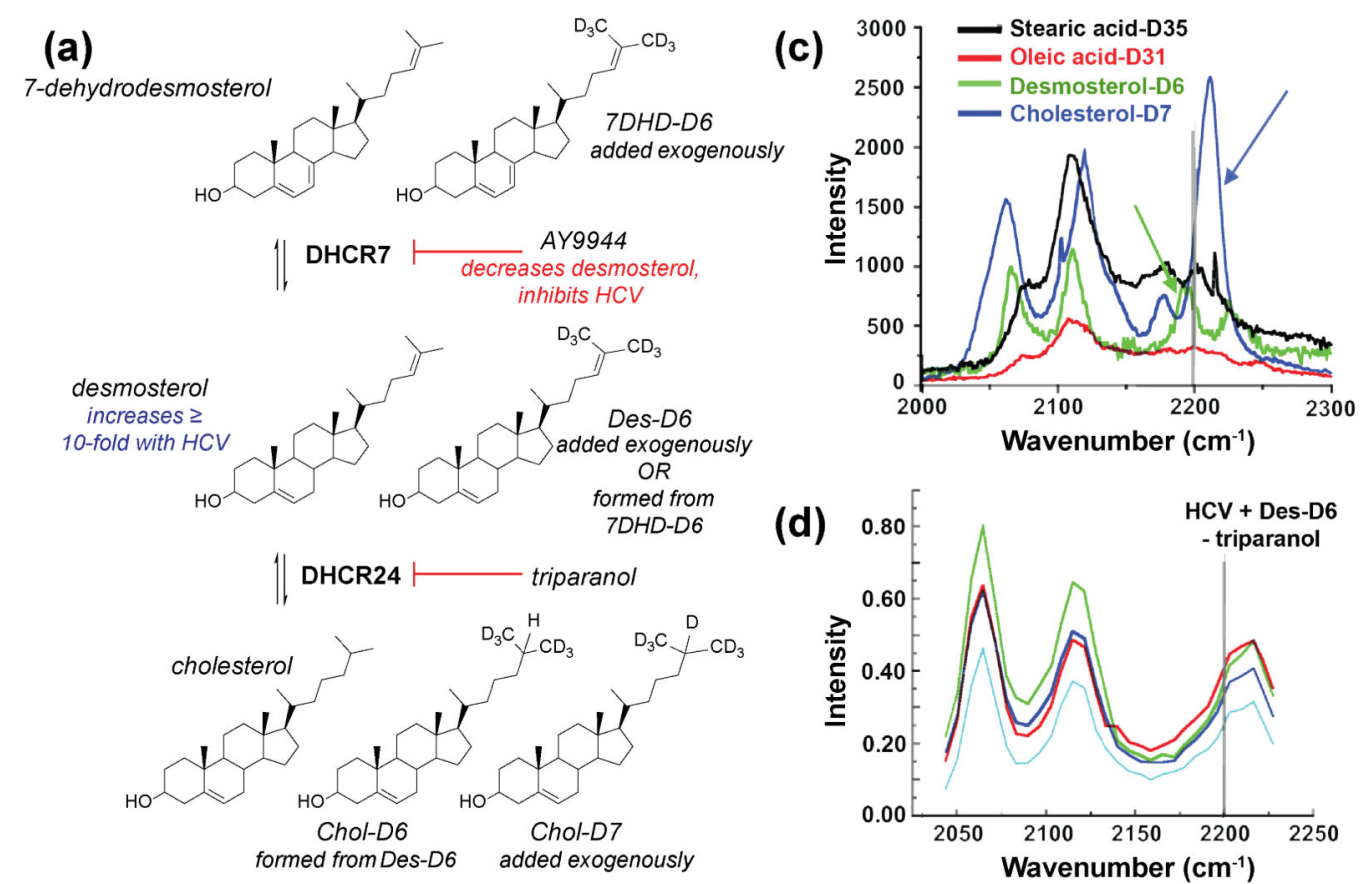

(b)
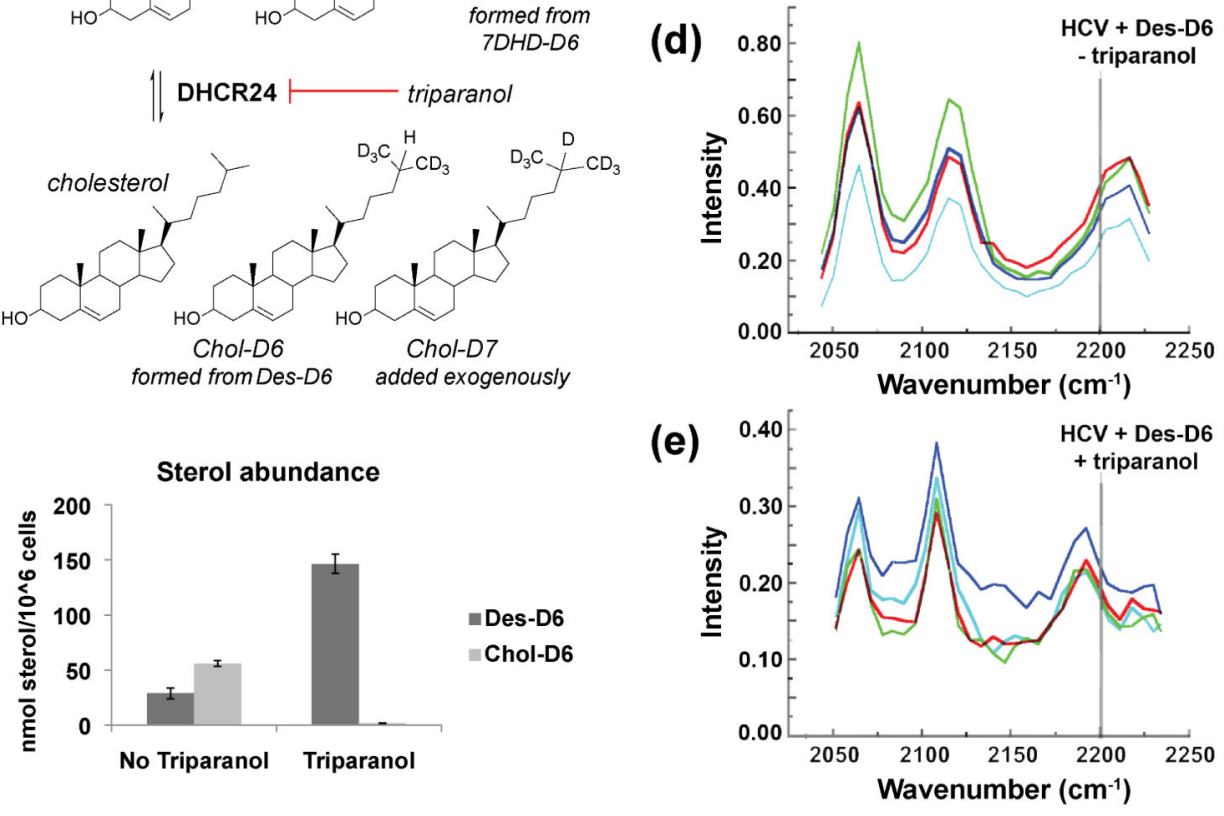

Figure 1. Conversion of exogenously added deuterated desmosterol to cholesterol is inhibited by triparanol

(a) Sterols and enzymes in late-stages of the Bloch pathway. HCV-JFH1 causes a ten-fold or greater increase in intracellular desmosterol. Deuterated substrates (Des-D6, 7DHD-D6, and Chol-D7) and inhibitors (AY9944, triparanol) are indicated. (b) LC-MS of extracted whole cell lipidomes. Lipids were extracted from Huh7.5 cells treated with triparanol and deuterated desmosterol (Des-D6). Whole-cell lipidomes were analyzed by LC-MS to quantify the abundance of Des-D6 and Chol-D6. Error bars represent the standard deviation of $n=2$ biological replicates. (c-e) Hyperspectral SRS microscopy wave number scans of (c) deuterated standards collected under cell-free conditions and of individual puncta in $\mathrm{HCV}$ JFH1-infected Huh7.5 cells treated with (d) Des-D6 alone or (e) Des-D6 with triparanol. For (c), the deuterated cholesterol (Chol-D7) standard has a peak at wave number greater than 2200 nm-1 (blue arrow) whereas the deuterated desmosterol standard has a peak at a lower wave number, less than $2200 \mathrm{~nm}-1$ (green arrow). For (d) and (e), each colored trace represents the wavelength scan of an individual punctum. The spectra obtained for puncta in HCV JFH1-infected cells treated with triparanol (e) is similar to the Raman spectrum of the desmosterol standard (c). For (c-e), each scan represents an analyzed punctum $(n>4)$. 
a

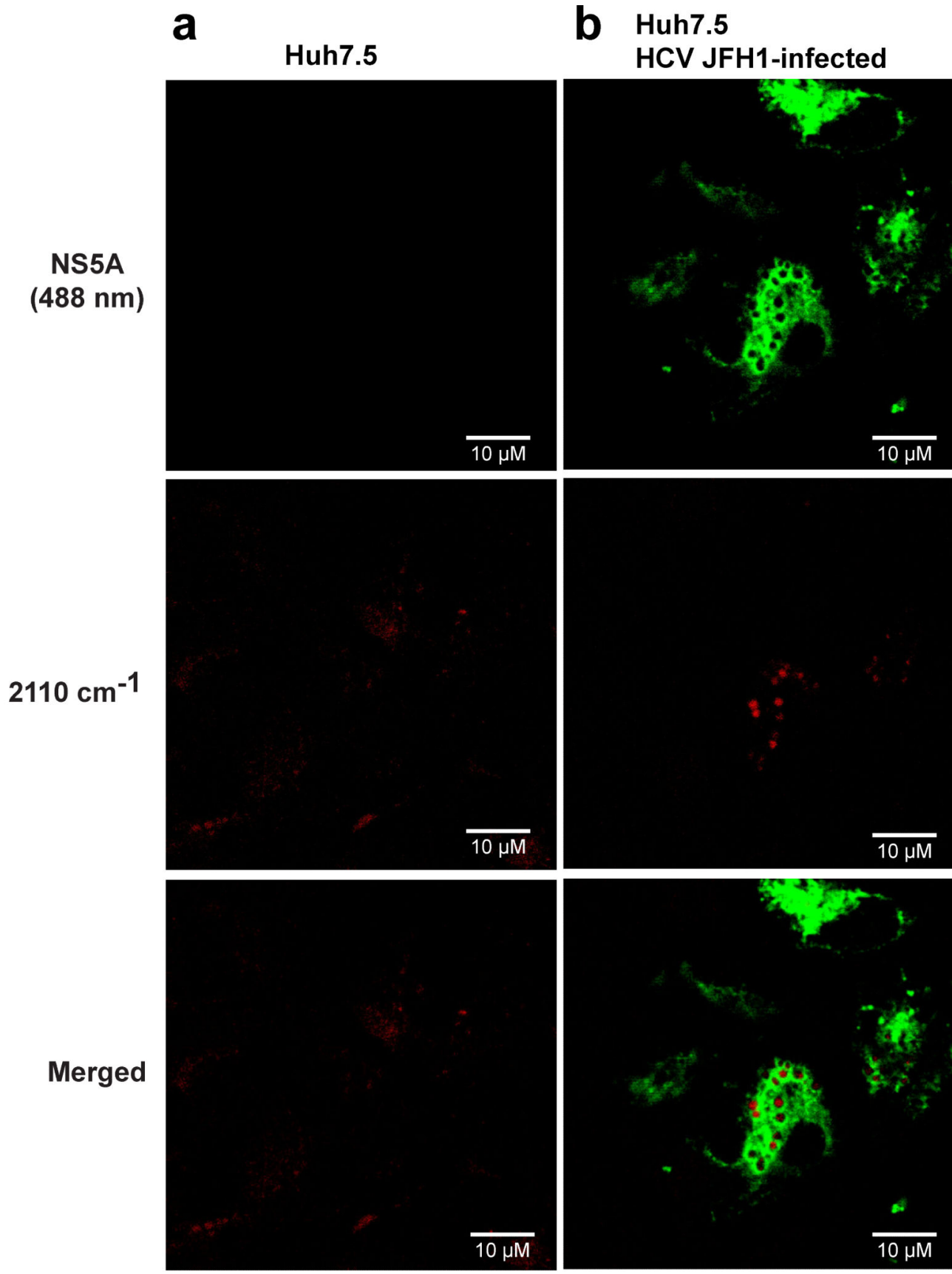

Figure 2. Deuterated desmosterol forms distinct puncta in HCV-infected Huh7.5 cells Huh7.5 cells were infected with HCV JFH1 or mock-infected and then treated with triparanol and exogenous deuterated desmosterol (Des-D6). 48 hours post-infection, cells were fixed, and immunofluorescence staining for the HCV NS5A protein was performed. SRS microscopy lasers were tuned to the C-D stretch $\left(2110 \mathrm{~cm}^{-1}\right)$ of deuterated desmosterol (Des-D6). Des-D6 and HCV viral protein, NS5A, are pseudocolored red and green, respectively. Representative images of (a) mock-infected and (b) HCV JFH1-infected 
Huh7.5 cells are presented from an experiment performed independently $n \geq 2$. Additional representative images are provided in Fig. S3. Scale bar equals 10 micrometers. 
a Huh7.5

+ Oleic acid

+ Des-H6
b Huh7.5
+ Oleic acid
+ Des-D6
C Huh7.5 HCV JFH1
+ Des-D6

C-H

$2854 \mathrm{~cm}^{-1}$
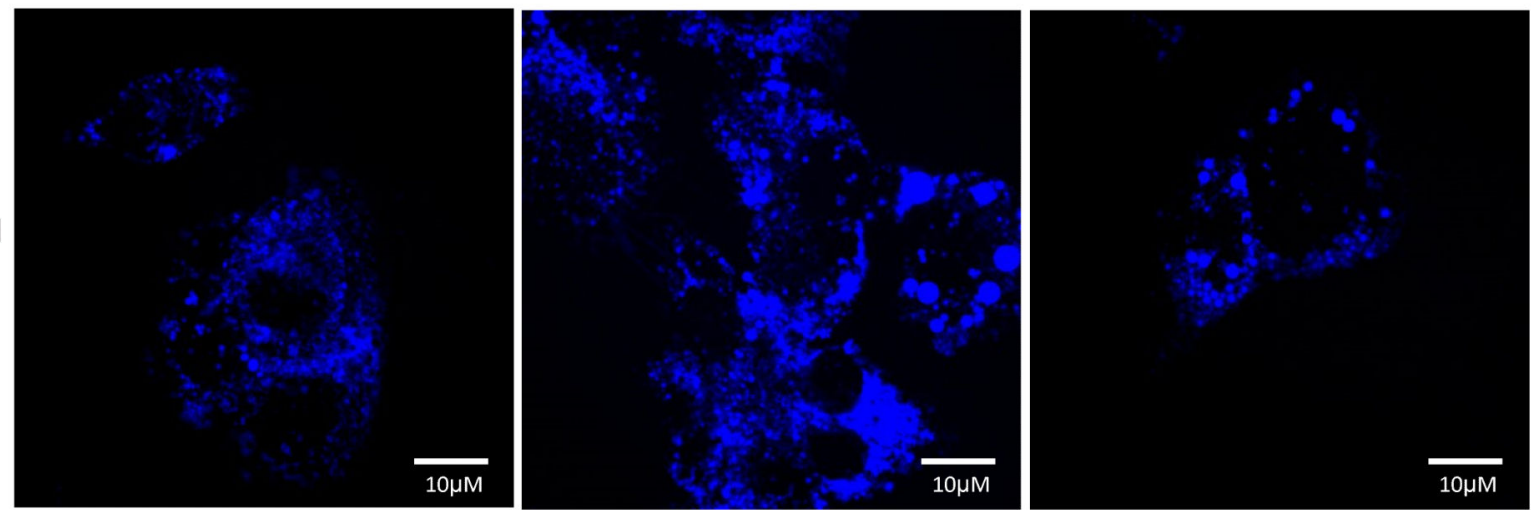

C-D

$2110 \mathrm{~cm}^{-1}$
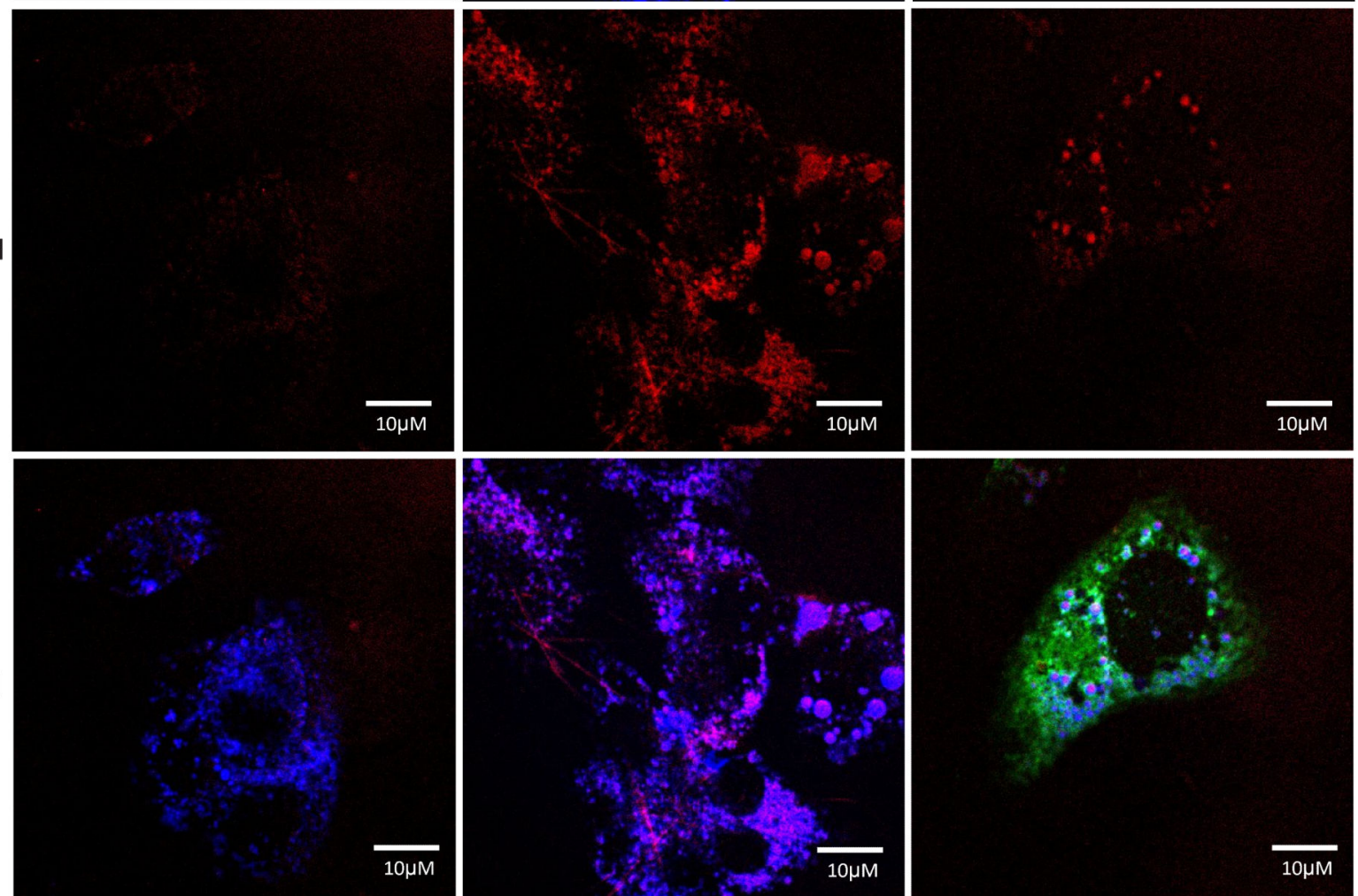

Figure 3. Des-D6 colocalizes with lipid droplets in cells treated with oleic acid or infected with HCV JFH1

Huh7.5 cells were treated with oleic acid (a, b) or infected with HCV-JFH1 (c) to induce lipid droplet formation. Cells were treated with $2 \mu \mathrm{M}$ triparanol and supplemented with exogenous Des-H6 (a) or Des-D6 (b, c). 48 hours post-infection, cells were fixed, and imaged. SRS microscopy lasers were tuned to the C-D stretch $\left(2110 \mathrm{~cm}^{-1}\right.$, pseudocolored red) of deuterated desmosterol (Des-D6) and to the C-H stretch $\left(2854 \mathrm{~cm}^{-1}\right.$, pseudocolored blue) present in endogenous lipids and also present in exogenously added Des-H6. Note that little or no signal is detected in cells supplemented with Des-H6 when the lasers are tuned to 
detect the C-D bond $\left(2110 \mathrm{~cm}^{-1}\right)$, demonstrating the selectivity of this imaging method for the C-D bond. Data shown are representative images from experiments that were independently performed $n=2$ times. Scale bar equals 10 micrometers. 

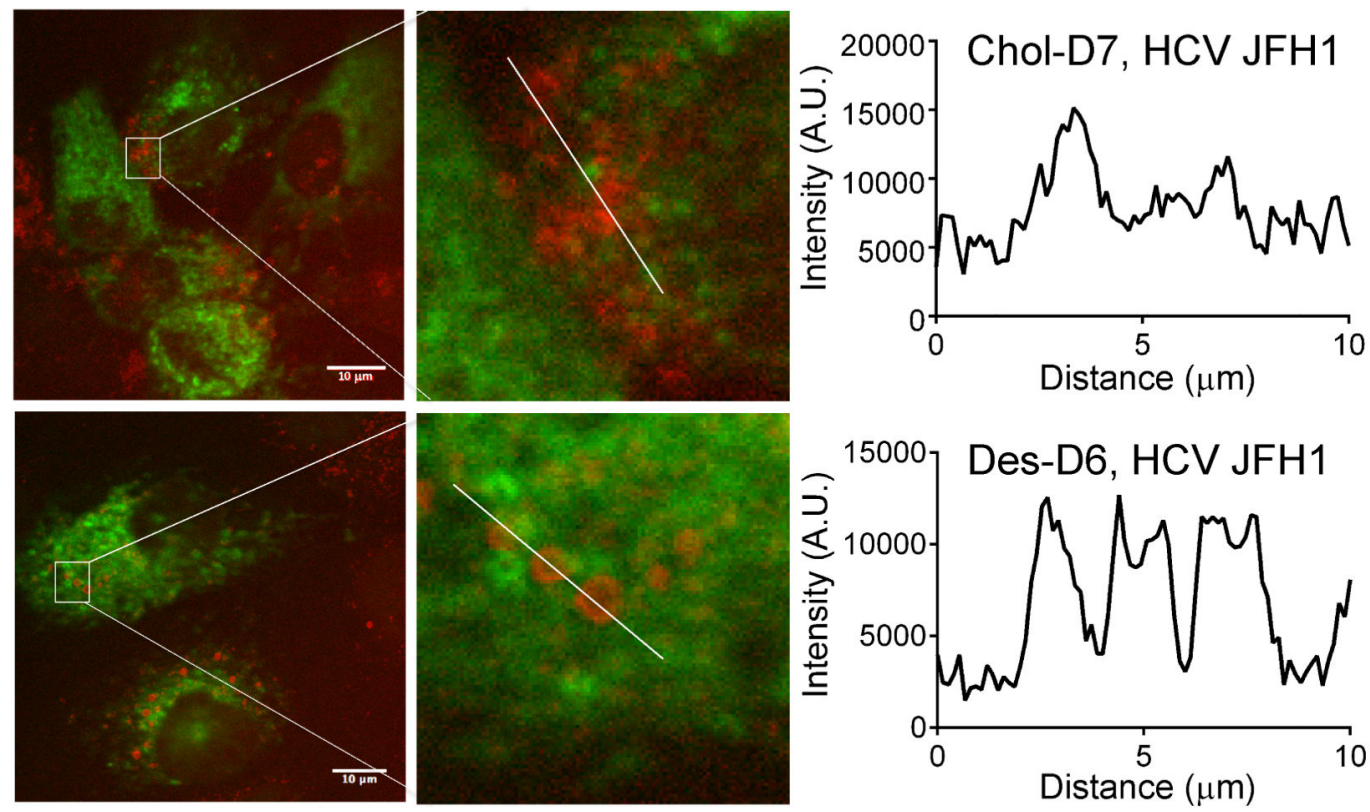

Figure 4. Des-D6 and Chol-D7 localized in lipid droplets with distinct visual phenotypes Huh7.5 cells were treated with triparanol, infected with HCV-JFH1, and supplemented with Chol-D7 or Des-D6 as indicated. At 48 hours post-infection, cells were fixed and imaged by SRS microscopy to visualize localization of the C-D bond stretch at $2110 \mathrm{~cm}^{-1}$ for Des-D6 and $2220 \mathrm{~cm}^{-1}$ for Chol-D7. Chol-D7 localizes in puncta that are smaller and that colocalize in amorphous clusters when compared to Des-D6. The number of wavenumber stacks imaged for each sample were identical, and the representative images shown correspond to a single image made from identical stack numbers. Note, the stacks described represent shift in wavenumber (see Fig. 1c), and do not represent imaging in the z-plane. C$\mathrm{D}$ intensity was plotted as function of distance in regions designated by white lines using ImageJ. Scale bar equals 10 micrometers. 